\title{
CONTROL OF THE SPATIAL STRUCTURE IN PRESCHOOL CHILDREN
}

\author{
N. Dimitrova* \\ Department of Foreign Languages and Information Technologies, National Sports Academy \\ "Vassil Levski", Sofia, Bulgaria
}

\begin{abstract}
In early childhood, most motor tasks are based on kinematic structure, as children assimilate motor culture in the way of imitation actions. Substantial development during this period also suffers from the controlling system, as a major problem is the differentiation of the effects of development on the one hand and education on the other. According to our working hypothesis, we assume that the educational process, both in primary school and pre-school age, should focus not on physical qualities but on improving the functioning of the controlling (external and internal circle) system. The aim of this study is to quantify some basic parameters for the management of spatial biomechanical characteristics in the earliest childhood. The selected test motor tasks registered regional and global accuracy assessment mutual location and the reachable area between cinematic pairs.
\end{abstract}

Key words: biomechanical characteristics, educational process, controlling system

\section{INTRODUCTION}

It is obvious the movement system is realized from the complex interaction among the cinematic, dynamic, different stages and the so called structures. In the early childhood most of the motor tasks are solved by using the cinematic structure because the children learn this kind of culture using the imitating actions (1-3).

Altogether with the biomechanical constants in the human body the controlling system is changing as well influenced by the evolution and the education. Certain partial researches from some authors /ex. Heizel, Thomson/ taking into consideration identical gemini and it seems the education does not play such an important role. In general the training interactions increase the motor opportunities; however those who are not trained catch up quite quickly/ within a few training sessions/ (4-8).

The practice proves the most important are the physical education methods. The

\footnotetext{
*Correspondence to: Nikolina Dimitrova, National Sports Academy "Vassil Levski", Department of Foreign Languages and Information Technologies, Studentski grad 1700 Sofia, Bulgaria, E-mail ninansa@abv.bg
}

biomechanics constant in the human body evolve as well.

The analysis and the literature sources indicated in the majority cases are registered and interpreted data are related to the physical qualities (9-14).

This side is quite variable and depends from several factors which are part of the routine life and could not be controlled.

According to our working hypothesis we suppose the educational process- the elementary school and preschool is oriented mainly to improve the activity of the controlling system /internal and external circle/system and just little part is devoted to the physical qualities. Due to those researches we found out the wrongly interpreted results received from the authors stating the most important part is the evolution one and not the education itself.
AIM
Obviously the basic aim of the present research was to assess the quantity of some basic Parameters for the control of the space biomechanical characteristics from earliest childhood. 


\section{METHODS}

In order to assess the spice parameters we have used video computer methodology and for the preparing of the data we used mathematic formalism for the variation and correlation statistical analyses.

The selected motor tasks for test have registered the regional and the global accuracy for mutual location in the reachable area among the different kinematic pairs.

\section{RESULTS}

In Table 1 the results related to the accuracy are presented when controlling the angle parameters in the shoulder joint. As a matter of fact the accuracy increases with the age; however as a general rule the errors are oriented in the negative direction. The control related to the accuracy is better with the smaller angle relationships up to 4 years of age. For all age groups and for the smaller angle relationships the standard deviations are greater.

Table 1. Cinematic pairs /shoulder joint// frontal plane /

\begin{tabular}{|c|c|c|c|c|c|c|l|}
\hline year & $\Delta \alpha 45 \%$ & $\mathrm{~S}$ & $\Delta \alpha 90 \%$ & $\mathrm{~S}$ & $\Delta \alpha 45 \%$ & $\mathrm{~S}$ & Remarks \\
\hline 3 & -26 & 20 & -18 & 10 & 22 & 12 & Compensation district \\
\hline 4 & -16 & 12 & -16 & 4 & 19 & 8 & Compensation district \\
\hline 5 & -8 & 10 & -2 & 6 & 8 & 7 & - \\
\hline 6 & 2 & 12 & 1 & 5 & 6 & 4 & - \\
\hline 7 & 3 & 8 & 3 & 5 & 5 & 4 & - \\
\hline
\end{tabular}

Obviously these compensating mechanisms play an important role in the controlling movement. As a matter of fact other observations prove this tendency remains for a long period of time and it is possible to be observed at some sports/ ex. Tennis/ and even with top athletes. And then the search the answer which is the methods and the means used to control the school-education process. It is interesting to notice the direction of the committed error depending from the initial posture. We are aware of the influence from the previous relations among the cinematic districts and it is proved with the transit data from greater to smaller angles. Particular interest represents the presence of compensating mechanisms in the distal districts being part of the cinematic chain.

We have assessed the global reachable zone by using a target and taking into consideration the errors in the frontal and the sagittal plane. The results are presented in Table 2 as for the 3 years old the test is not always right. $/ \mathrm{R}<0,5 /$. In the frontal plane the notes are received thanks to a target, and in the sagittal one by using video meters in centimetres.

Table 2. Reachable area

\begin{tabular}{|l|l|l|l|l|l|l|}
\hline years & \multicolumn{4}{|l|}{ Boys } & \multicolumn{3}{l|}{ Girls } \\
\hline & $\Delta \gamma$ & $\mathrm{S}$ & $\mathrm{V}$ & $\Delta \gamma$ & $\mathrm{S}$ & $\mathrm{V}$ \\
\hline 4 & 3,1 & 0,8 & 25.8 & -48 & 16 & $-33,33$ \\
\hline 5 & 3,4 & 1,4 & 41,17 & -29 & 15 & $-51,72$ \\
\hline 6 & 6,1 & 1,1 & 18,03 & -34 & 12 & $-35,29$ \\
\hline 7 & 5,9 & 0,8 & 13,55 & 26 & 11 & $-42,30$ \\
\hline
\end{tabular}

The improvement in the frontal plane done between 4 and 5 years of age and later one between 5 and 6 years of age is accomplished with a quality jump with some stagnation afterwards. The whole motor task supposes global moving of the Gravity Centre and those data would rather interpret the regional accuracy into the shoulder joint itself. This joint has got three levels of freedom and the future studies will assess the accuracy and the sensitivity for each level. In our case in order to unite the test, the target was placed on a height requiring to lift the arm up to 60 degrees: the height varies depending on how tall is the human body.

The accuracy in the sagittal plane indicates two important results

a) all values are negative and

б) a local minimum is observed at 5 years old. Our explanation of this anomaly /worsening of the results at the 6 years old/ is related to the psychological factors when children think to not go beyond the defined aim itself. Finally these results are not enough and we should take it into consideration during the schooleducational process. Perhaps we recognize the well-known self- conservation instinct in the sport biomechanics.

\section{CONCLUSION}

According to the received results we present the following conclusions and recommendations:

1. The data analyses from the experiment have established the presence of legal importance 
and as a result the compensating mechanisms are directed to the local activities from the global one and through the regional one. All those analyses could be discovered in the mature age and is good to overcome the negative influences during the training education methods.

2. When we compare the accuracy to adults during the performance of motor tasks it is strongly influenced from the previous postures and spatial characteristics. This fact should also be taken into consideration in the schooleducational process.

3. Because of the rapid development of the motor system more tests should be prepared according to the different age groups mostly for children 3 and 4 years of age. The longitude study should be supported and adapted to the appropriate tests within the age. 4. The time development in the motor system during the preschool age differs: we have having stagnation stage and quality jumps as well. More important studies are needed in order to find out the relations related to the development of the rest functional systems and to determine the efficiency, the right direction for the school-educational process and its stages.

5. An important conclusion for the training is the fact that the legal systematic error related to the studied accuracy in the sagittal plane is also observed.

\section{REFERENCES}

1. Ivanov, Sv., Arakchijski, Zdr., Dimitrova, N., Specificity of motor culture assessments in preschool children; Sports, Society, Education, NSA Press, Sofia, 2:36-42, 1996.

2. Gikova, M., Ivanov, Sv., Dimitrova, N., Traykova, Bl., Zareva, I., Assessment of some parameters of management of strength structure of motor system., Sport, Society, Education, NSA Press, Sofia, 3: 111-115, 2000.

3. Pendeva, M., Ivanov, Sv., Dimitrova, N., Frequency stability characteristics, Sport, Society, Education., NSA Press, Sofia, 4: 220-226, 2001.
4. Popova, N., Grueva-Pancheva, T., Investigation of the physical capacity of students from the secondary schools in Veliko Tarnovo, Annual of the National Sports Academy "Vasil Levski", NSA PRESS, 1: 34-38, 2019.

5. Popova, N., Features of the physical capacity of students from 1st to 4th grade from Veliko Tarnovo; Annual of the National Sports Academy "Vasil Levski, NSA PRESS, 1: 98-104, 2019.

6. Kostova, N., State of Physical Capacity in Pupils from Primary School, Annual of NSA "Vasil Levski", 1: 73-81, 2019.

7. Kostova, N., Tsonkova, P., Analysis of sports equipment management at the secondary schools, International Scientific Congress "Applied Sports Sciences", 432437, 2019.

8. Iliev, I., Nikolov, N., Specifics of Physical Training in Wrestling Wrestling., Department of Fighting and Judo, Sport and Science, extraordinary issue 4: 17-19, 2013.

9. Avramov, E., Avramova, M., Dependencies between the anthropometric indicators and the sport-technical characteristics of the teenage handlers (12 years). Sports and science, 2 :57-60, 2017.

10.Albert, M., Study of the health condition of practice dentistries, rehabilitators and massage; Trakia Journal of Sciences, 17 (1), 826-831, 2019.

11.Nikolova, A., Frequency of injuries among elite bulgarian athletes; Trakia Journal of Sciences, 17, (1), 868-872, 2019.

12.Nikolova, A., Study of Injuries in Martial Arts Proceeding Book, "Applied Sports Sciences", 323-328, 2019.

13. Yaneva, A., Miletiev, S., Fitness education for students in the discipline "sport, Trakia Journal of Sciences, 17 (1), 723-730, 2019;

14. Yaneva, A., Lukanova, V., „Examining the opinion of experts in judo on the need for a training methodology for children aged 7 to 10"; Trakia Journal of Sciences, 17 ( 1), 731-735, 2019. 